\title{
ON THE NULLITY AND ENCLOSURE GENUS OF WILD KNOTS
}

\author{
BY \\ JAMES M. MCPHERSON( $\left.{ }^{1}\right)$
}

The fundamental group of the complement of a wild knot in a 3-sphere can be expressed as the colimit (direct limit) of a suitable family of groups and homomorphisms (Crowell [4]). To each group in the family we assign a Jacobian module, and in $\S 1$ we prove that this assignment is functorial and preserves colimits. This is used in $\$ 2$ to show that the nullity of the Alexander module of a knot with one wild point is bounded above by its enclosure genus. This can be used in some cases to calculate the enclosure genus and the penetration index of the knot in a purely algebraic way. In $\$ 3$ we give examples to show that the upper bound of Theorem 2 is the best possible, along with some results concerning the penetration index of an arc relative to surfaces of genus $r$.

\section{The Alexander module.}

1. For the categorical notions used here, see [10]. The proof of Theorem 1 will depend on the following standard result ([10, Chapter II, 6.2]):

(1.1) Let $\mathscr{A}$ be a category with coproducts and let $\mathscr{B}$ be any category. Then the functor $T: \mathscr{A} \rightarrow \mathscr{B}$ is colimit preserving iff it preserves coproducts and coequalisers.

Let $H$ be an arbitrary group which will remain fixed; we define a category $\mathscr{H}$ of $H$-groups [ $G, \alpha]$ (cf. [8]) where $G \in \mathscr{G}$ (the category of all groups and homomorphisms), and $\alpha: G \rightarrow H$ is an epimorphism. A morphism $\theta:\left[G_{1}, \alpha_{1}\right] \rightarrow\left[G_{2}, \alpha_{2}\right]$ is a group homomorphism $\theta: G_{1} \rightarrow G_{2}$ such that the diagram

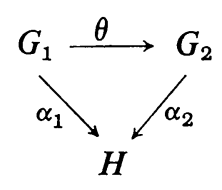

commutes, i.e. $\alpha_{2} \theta=\alpha_{1}$.

(1.2) $\mathscr{H}$ admits coproducts.

Proof. In $\mathscr{G}$, the coproduct of $G_{1}$ and $G_{2}$ is just the free product $G_{1} * G_{2}$. By the universality of the coproduct diagram in $\mathscr{G}[9$, p. 17], there exists a unique map

Received by the editors September 18, 1968.

(1) Research done at the University of New South Wales where the author was supported by an Australian Commonwealth Postgraduate Research Scholarship. The author wishes to thank Professor N. F. Smythe for his many helpful suggestions. 
$\alpha: G_{1} * G_{2} \rightarrow H$ making the diagram

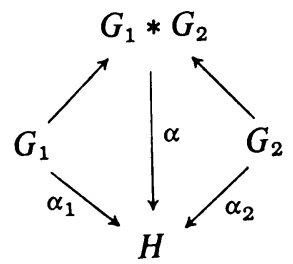

commute.

Since the $\alpha_{i}$ are epimorphisms, $\alpha$ is an epimorphism and $\left[G_{1} * G_{2}, \alpha\right]$ is an $H$-group which is the coproduct in $\mathscr{H}$ of $\left[G_{1}, \alpha_{1}\right]$ and $\left[G_{2}, \alpha_{2}\right]$.

(1.3) $\mathscr{H}$ admits coequalisers.

Proof. In $\mathscr{G}$, the coequaliser of $\theta_{1}, \theta_{2}: G \rightarrow L$ is the canonical map $\tau: L \rightarrow L / \Theta$, where $\Theta$ is the normal subgroup of $L$ generated by all elements of the form $\theta_{1}(g) \theta_{2}\left(g^{-1}\right)$ for $g \in G$.

For $H$-groups $[G, \alpha]$ and $[L, \beta]$, since $\theta_{1}$ and $\theta_{2}$ are $H$-morphisms, we have $\beta \theta_{1}=\alpha=\beta \theta_{2}$. Then in $\mathscr{G}, \beta$ factors uniquely through the coequaliser of $\theta_{1}$ and $\theta_{2}$, i.e. $\beta=\sigma \tau$ for a unique $\sigma$; but $\beta$ is an epimorphism, so $\sigma$ is also. It follows that $\tau:[L, \beta] \rightarrow[L / \Theta, \sigma]$ is an $H$-morphism and is the coequaliser in $\mathscr{H}$ of the maps $\theta_{1}$ and $\theta_{2}$.

2. Let $[G, \alpha] \in \mathscr{H}$ and suppose $K=\operatorname{ker} \alpha$, i.e. the sequence

$$
1 \longrightarrow K \longrightarrow G \stackrel{\alpha}{\longrightarrow} H \longrightarrow 1
$$

is exact. Then we have an exact sequence

$$
0 \longrightarrow K / K^{\prime} \longrightarrow G / K^{\prime} \stackrel{\alpha}{\longrightarrow} H \longrightarrow 1
$$

with abelian kernel, where $\alpha: G / K^{\prime} \rightarrow H$ is induced by $\alpha$. By [5], we have a uniquely determined exact sequence of $J H$-modules

$$
0 \longrightarrow K / K^{\prime} \longrightarrow H_{1}(G, 1: J H) \stackrel{\alpha}{\longrightarrow} J H \stackrel{\varepsilon}{\longrightarrow} J \longrightarrow 0
$$

where the relative homology group $H_{1}(G, 1: J H)$ has the Jacobian matrix of $G$ evaluated at $\alpha$ (see [3]) as a relation matrix over the integer group ring $J H$ of $H$, and $\varepsilon: J H \rightarrow J$ is the trivialiser.

Alternatively, we may use MacLane's statement [9, p. 120] of the same sequence

$$
0 \longrightarrow K / K^{\prime} \longrightarrow M_{\alpha} \stackrel{\alpha^{*}}{\longrightarrow} J H \longrightarrow J \longrightarrow 0
$$

where $M_{\alpha}$ is the $J H$-module with generators $[g]$ for $g \in G / K^{\prime}$, and relations [1]= $0_{2}\left[g_{1} g_{2}\right]=\alpha\left(g_{1}\right)\left[g_{2}\right]+\left[g_{1}\right]$. The map $\alpha^{*}$ is defined by $\alpha^{*}[g]=\alpha(g)-1$.

It follows from Trotter's work [11] that $M_{\alpha}$ is naturally isomorphic to

$$
H_{1}(G, 1: J H) \text {. }
$$


We say that $M_{\alpha}=M[G, \alpha]$ is the Jacobian module of $G$ evaluated at $\alpha$; if $H=G / G^{\prime}$ and $\alpha$ is the abelianiser $\alpha: G \rightarrow G / G^{\prime}, M_{\alpha}$ is called the Alexander module of $G$. When there is no danger of confusion, we write $M G$ for $M[G, \alpha]$. In this paragraph we will mainly be using MacLane's definition of the Jacobian module, and in $\$ 2$ we will use the relative homology definition.

3. Let $\theta:[G, \alpha] \rightarrow[L, \beta]$ be an $H$-morphism; then we have induced maps of exact sequences

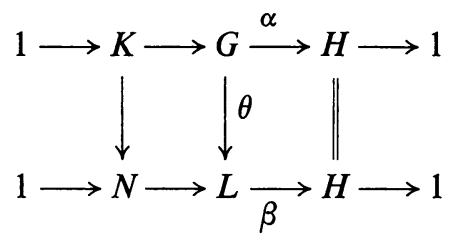

and

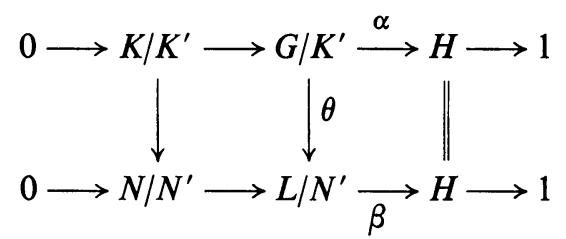

We define a map $M \theta: M[G, \alpha] \rightarrow M[L, \beta]$ by $(M \theta)\left[g K^{\prime}\right]=\left[\theta(g) N^{\prime}\right]$ for $g \in G$, and linearity. Obviously $M \theta\left[K^{\prime}\right]=\left[\theta(1) N^{\prime}\right]=0$ and $M \theta\left[g_{1} g_{2} K^{\prime}\right]=\alpha\left(g_{1} K^{\prime}\right) M \theta\left[g_{2} K^{\prime}\right]$ $+M \theta\left[g_{1} K^{\prime}\right]$ since $\beta \theta=\alpha$ and $\alpha\left(K^{\prime}\right)=1$. Thus $M \theta$ is a properly defined $J H$-morph ism.

Moreover, $M 1=1$ and $M(\theta \phi)=M \theta M \phi$, so if $\mathscr{M}$ is the category of $J H$-modules, $M: \mathscr{H} \rightarrow \mathscr{M}$ is a functor, called the Jacobian module functor.

THEOREM 1. The Jacobian module functor preserves colimits.

Proof. (i) $M$ preserves coproducts.

For, let $G$ have presentation $\left(x_{i}: r_{i}\right), i=1,2$. Then $G_{1} * G_{2}$ has presentation $\left(\boldsymbol{x}_{1} \cup \boldsymbol{x}_{2}: \boldsymbol{r}_{1} \cup \boldsymbol{r}_{2}\right)$ and $H_{1}\left(G_{1} * G_{2}, 1: J H\right)$ has a relation matrix of the form

$$
\left|\begin{array}{cc}
A_{1} & 0 \\
0 & A_{2}
\end{array}\right|
$$

where $A_{i}$ is a relation matrix for $H_{1}\left(G_{i}, 1: J H\right)$. Thus

$$
H_{1}\left(G_{1} * G_{2}, 1: J H\right) \cong H_{1}\left(G_{1}, 1: J H\right) \oplus H_{1}\left(G_{2}, 1: J H\right) .
$$

(ii) $M$ preserves coequalisers.

Let $\tau:[L, \beta] \rightarrow[L / \Theta, \sigma]$ be the coequaliser of $\theta_{1}, \theta_{2}:[G, \alpha] \rightarrow[L, \beta]$, as in the proof of (1.3). We want to prove that the Jacobian module of $[L / \Theta, \sigma]$ is the quotient of the Jacobian module of $[L, \beta]$ by the submodule generated by all elements of the form $M \theta_{1}(m)-M \theta_{2}(m)$ for $m \in M G$. It is sufficient to show that this submodule contains $\operatorname{ker} M \tau$, since $M \theta_{1}(m)-M \theta_{2}(m) \in \operatorname{ker} M \tau$ for all $m$. 
So let $\left[l N^{\prime}\right] \in \operatorname{ker} M \tau$; then $\left[\tau\left(l N^{\prime}\right)\right]=\left[\tau(l) P^{\prime}\right]=0$, where $P=\operatorname{ker} \sigma: L / \Theta \rightarrow H$. Thus $\tau(l) \in P^{\prime}$, and so $l \in N$, since $\beta(l)=\sigma \tau(l)=0 . \tau: N^{\prime} \rightarrow P^{\prime}$ is onto, and $\tau(l) \in P^{\prime}$, so $l=W l_{1}$ where $l_{1} \in N^{\prime}$ and $W \in \operatorname{ker} \tau$. Therefore $\left[l N^{\prime}\right]=\left[W N^{\prime}\right]$, and since $W \in \operatorname{ker} \tau, W$ can be written

$$
W=\prod_{j} l_{j}^{e_{j}} \theta_{1}\left(g_{j}\right) \theta_{2}\left(g_{j}^{-1}\right) l_{j}^{-e_{j}}
$$

for $g_{j} \in G$. Using the relations

$$
\left[l^{-1}\right]=-\beta\left(l^{-1}\right)[l]
$$

and

$$
\left[l^{m}\right]=\sum_{j=0}^{m-1} \beta\left(l^{j}\right)[l]
$$

for $l \in L / N^{\prime}$, it follows by direct calculation that

$$
\left[l N^{\prime}\right]=\sum_{j} \beta\left(l_{j}^{l_{j}}\right)\left\{M \theta_{1}\left[g_{j}\right]-M \theta_{2}\left[g_{j}\right]\right\}
$$

as required.

Thus $M$ preserves coproducts and coequalisers, and the result follows from (1.1). Q.E.D.

4. Let $R$ be an integral domain and let $M$ be a finitely presented $R$-module $(\boldsymbol{X} ; \boldsymbol{P})$ where $\rho_{i} \in \boldsymbol{P}$ is a relation of the form

$$
\rho_{i}=\sum_{j=1}^{m} \alpha_{i j} x_{j}, \quad x_{j} \in X, \alpha_{i j} \in R .
$$

The matrix $\left(\alpha_{i j}\right)$ is a relation matrix for the module $M$. Let $A$ be a finite matrix over $R$, with $m(A)$ columns and rank $r(A)$; we define the nullity $\nu(A)$ of $A$ to be the difference $m(A)-r(A)$. It is easy to see that this definition is equivalent to that given in [3]. If $A$ is a relation matrix for the module $M$, we say that $M$ has nullity $\nu(A)$, since the Tietze operations on matrices preserve nullity. If $M$ is infinitely presented and $R$ is Noetherian, we take a sequence $\left\{M_{i}\right\}$ of finitely presented submodules of $M$, such that $M_{i} \subset M_{i+1}$ and $\bigcup_{i} M_{i}=M$, and define

$$
\nu(M)=\sup \nu\left(M_{i}\right) \text {. }
$$

(4.1) Let $A=\left(\alpha_{i j}\right)$ and $B=\left(\beta_{k j}\right), j=1, \ldots, m$, be finite matrices over $R$. If $A$ has $l$ rows, the matrix $C=\left(\gamma_{i j}\right)$ defined by

$$
\begin{aligned}
\gamma_{i j} & =\alpha_{i j}, & & i \leqq l, \\
& =\beta_{i-l, j}, & & i>l,
\end{aligned}
$$

has nullity at least equal to $\nu(A)-r(B)$.

Proof. Immediate since $r(C) \leqq r(A)+r(B)$.

(4.2) Let $0 \rightarrow A \rightarrow B \rightarrow C \rightarrow 0$ be a short exact sequence of $R$-modules, and suppose $A$ is generated by $\left\{n_{1}, \ldots, n_{s}\right\}$. Then $\nu(B)-s \leqq \nu(C) \leqq \nu(B)$. 
Proof. $B$ can be represented as a quotient of a free module $F$; let $\phi: F \rightarrow B$ be the presentation map. $A$, as a submodule of $B$, is generated by $s$ elements of the form $\rho_{i}=\sum_{j} \alpha_{i j} \phi\left(x_{j}\right)$ if $F$ is free on the alphabet $\left\{x_{j}\right\}$. But $\sum \alpha_{i j} \phi\left(x_{j}\right)=\phi\left(\sum \alpha_{i j} x_{j}\right)$, so $C \cong B / A$ means that if we add the matrix $\left(\alpha_{i j}\right), i=1, \ldots, s$, to some relation matrix for $B$, we obtain a relation matrix for $C$. The results then follow from (4.1) and $[3,2.2]$.

Let $\left[G_{i}, \alpha_{i}\right]$ be $H$-groups, $i=0,1,2$, and suppose $G_{0}$ is a free group on $m$ generators. If $G$ is the colimit of the system

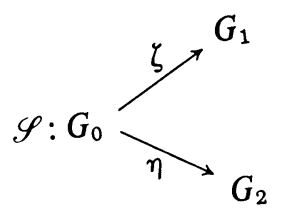

where $\zeta$ and $\eta$ are $H$-morphisms, then $[G, \alpha]$ is an $H$-group for a unique $\alpha: G \rightarrow H$, since $H$ admits coproducts and coequalisers. In fact, if $w_{i}: G_{i} \rightarrow G_{1} * G_{2}$, then $G$ is the quotient of $G_{1} * G_{2}$ by the normal subgroup $\left\langle\left\langle w_{1} \zeta(g) w_{2} \eta\left(g^{-1}\right): g \in G_{0}\right\rangle\right\rangle$. Since the Jacobian module functor preserves colimits, $M[G, \alpha]$ is the quotient of $M\left[G_{1}, \alpha_{1}\right] \oplus M\left[G_{2}, \alpha_{2}\right]$ by the submodule $K$ generated by all elements of the form $M w_{1} \zeta[g]-M w_{2} \eta[g]$ for $[g] \in M\left[G_{0}, \alpha_{0}\right]$. The elements $M w_{1} \zeta\left[x_{i}\right]-M w_{2} \eta\left[x_{i}\right]$, $i=1,2, \ldots, m$, generate $K$ if $G_{0}$ is free on $x_{1}, \ldots, x_{m}$; thus $K$ is generated by $r \leqq m$ elements.

The sequence

$$
0 \rightarrow K \rightarrow M G_{1} \oplus M G_{2} \rightarrow M G \rightarrow 0
$$

is exact, so

$$
\nu\left(M G_{1} \oplus M G_{2}\right)-m \leqq \nu\left(M G_{1} \oplus M G_{2}\right)-r \leqq v(M G) .
$$

But $M G_{1} \oplus M G_{2}$ has a relation matrix of the form

$$
\left|\begin{array}{cc}
A_{1} & 0 \\
0 & A_{2}
\end{array}\right|
$$

so $\nu\left(M G_{1} \oplus M G_{2}\right)=\nu\left(M G_{1}\right)+\nu\left(M G_{2}\right)$. Hence,

$$
\nu\left(M G_{1}\right) \leqq \nu(M G)+m-\nu\left(M G_{2}\right) .
$$

2. Nullity and enclosure genus.

5. Our "knot" is an oriented knot in a 3-sphere $S$, with one wild point $w$. The extension to knots with finitely many wild points is easily accomplished.

A "handlebody (Henkelkorper) of genus $g$ " is a 3-cell with $g$ oriented handles. A sequence $H_{0} \supset H_{1} \supset H_{2} \supset \ldots$ of handlebodies is an admissible sequence for the knot $k$ if

(i) $H_{i}$ is a tame closed neighbourhood of $w$, for each $i$;

(ii) $H_{i+1} \subset$ Int $H_{i}$ for all $i$, and $\bigcap H_{i}=k$;

(iii) $k$ does not meet the boundary of any of the $H_{i}$. 
The enclosure genus of $k$ is then defined to be min lim genus $\left(H_{i}\right)$, where the minimum is taken over all admissible sequences $\left\{H_{i}\right\}$. We will denote the enclosure genus of $k$ by $e(k)$.

We choose an admissible sequence for $k$ which is of mimimal genus (i.e. genus $\left(H_{i}\right)=e(k)$ for all $i$ and set $G_{i}=\pi_{1}\left(S-H_{i}\right)$, and $G=\pi_{1}(S-k)$. Then the inclusions $S-H_{i} \subset S-H_{i+1}$ and $S-H_{i} \subset S-k$ induce maps $\phi_{i}: G_{i} \rightarrow G_{i+1}$ and $\psi_{i}: G_{i} \rightarrow G$, with $\psi_{i+1} \phi_{i}=\psi_{i}$.

Let $\mathfrak{a}: \mathscr{G} \rightarrow \mathscr{A}$ (the category of abelian groups) be the abelianisation functor, given by $\mathfrak{a}(G)=G / G^{\prime}$. We note that $\mathfrak{a}\left(G_{i}\right) \cong H_{1}\left(S-H_{i}\right)$ and $\mathfrak{a}(G) \cong H_{1}(S-k)$, so we have a commuting diagram

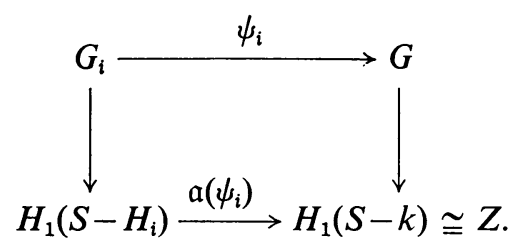

Now $\mathfrak{a}\left(\psi_{i}\right)$ is an epimorphism, and so is the map $G_{i} \rightarrow H_{1}\left(S-H_{i}\right)$, so we make $G_{i}$ a $Z$-group $\left[G_{i}, \alpha_{i}\right]$ by taking for $\alpha_{i}$ the composition of $\psi_{i}$ with the canonical map $\tau: G \rightarrow G / G^{\prime}$ and the Hurewicz isomorphism $G / G^{\prime} \rightarrow H_{1}(S-k)$. The composition of these last two maps will serve for our map $\alpha: G \rightarrow Z$, making $[G, \alpha]$ a $Z$-group. Then the maps $\phi_{i}:\left[G_{i}, \alpha_{i}\right] \rightarrow\left[G_{i+1}, \alpha_{i+1}\right]$ and $\psi_{i}:\left[G_{i}, \alpha_{i}\right] \rightarrow[G, \alpha]$ are morphisms in the category $\mathscr{Z}$ of $Z$-groups. In fact,

(5.1) $[G, \alpha]$ is the colimit in $\mathscr{Z}$ of the sequence

$$
\cdots \longrightarrow\left[G_{i}, \alpha_{i}\right] \stackrel{\phi_{i}}{\longrightarrow}\left[G_{i+1}, \alpha_{i+1}\right] \longrightarrow \cdots .
$$

Proof. By [4], $G$ is the colimit in $\mathscr{G}$ of the sequence $\cdots \rightarrow G_{i} \stackrel{\phi_{i}}{\rightarrow} G_{i+1} \rightarrow \cdots$. Suppose $[H, \beta]$ is the colimit in $\mathscr{Z}$ of the sequence of $Z$-groups. Since each $Z$-map is a group homomorphism, and $G$ is the colimit, there exists a unique map $G \rightarrow H$ in $\mathscr{G}$. By the same token, since $[G, \alpha]$ and the maps $\psi_{i}:\left[G_{i}, \alpha_{i}\right] \rightarrow[G, \alpha]$ form a commuting system of $Z$-groups, there exists a unique $Z$-map $[H, \beta] \rightarrow[G, \alpha]$. There is thus a unique map in $\mathscr{G}$ from $H \rightarrow G-$ so $G \cong H$ and $[G, \alpha] \cong[H, \beta]$.

From (5.1) and Theorem 1, we see that the Alexander module of $G$ is the colimit of the Jacobian modules of the $G_{i}$, evaluated at $\alpha_{i}$.

Consider the sequence

$$
\cdots M \psi_{i}\left(M G_{i}\right) \subset M \psi_{i+1}\left(M G_{i+1}\right) \cdots
$$

of finitely presented $J Z$-modules; there is a unique (inclusion) map $\bigcup M \psi_{i}\left(M G_{i}\right)$ $\subset M G$, and a unique map $M G \rightarrow \bigcup M \psi_{i}\left(M G_{i}\right)$, since each diagram

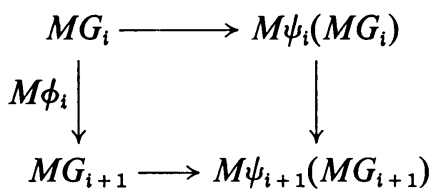


commutes, and $M G$ is the colimit of the $M G_{i}$. Hence $M G \cong \bigcup M \psi_{i}\left(M G_{i}\right)$, and from $([3], 2.2)$ we have

$$
\nu(M G) \leqq \sup v\left(M G_{i}\right) .
$$

6. It is known that the Alexander module of any knot has nullity at least one, and that the nullity of a tame knot is equal to one $[3,4.5,4.6]$.

THEOREM 2. Let $k$ be a knot in a 3-sphere $S$, with one wild point. If $\nu$ is the nullity of the Alexander module of $\pi_{1}(S-k)$, then $1 \leqq \nu \leqq e(k)$.

Proof. To prove the theorem, it is sufficient to show that $\nu\left(M G_{i}\right) \leqq e(k)$ for all $i$.

$H_{i}$ can be written as a 3-cell $U$ and $e(k)$ handles $h_{1}, \ldots, h_{e} . S-H_{i}$ is of the same homotopy type as the complement of the (tame) 3-cell $U$ and $e$ arcs $\alpha_{1}, \ldots, \alpha_{e}$ obtained by shrinking the solid handles of $H$ to $\operatorname{arcs}$ from $\operatorname{Bd} U$ to $\operatorname{Bd} U$.

We orient the $\operatorname{arcs} \alpha_{1}, \ldots, \alpha_{e}$ and join the $2 e$ points of $\operatorname{Bd} U \cap \bigcup \alpha_{i}$ by $e$ arcs $\beta_{1}, \ldots, \beta_{e}$ in Int $U$ so that $\bigcup\left\{\alpha_{i} \cup \beta_{i}\right\}$ is a tame knot $k^{*}$. Thickening $U$ appropriately, so we can apply the van Kampen theorem, we see that $\pi_{1}\left(S-k^{*}\right)$ is the colimit of the system

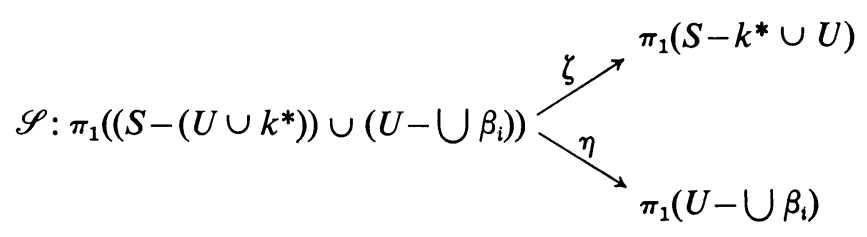

where $\zeta$ and $\eta$ are induced by inclusion. But $\left(\left(S-\left(U \cup k^{*}\right)\right) \cap\left(U-\cup \beta_{i}\right)\right)$ is of the same homotopy type as a 2 -sphere with $2 e$ oriented holes, and so has fundamental group free on $2 e-1$ generators. Moreover, we can choose the $\beta_{i}$ so they are unknotted and do not link each other; then $\pi_{1}\left(U-\bigcup \beta_{i}\right)$ is a free group on $e$ generators. From subsection 4 , we see that

$$
\nu\left(M G_{i}\right) \leqq 1+(2 e(k)-1)-\nu\left(M \pi_{1}\left(U-\bigcup \beta_{i}\right)\right)=e(k) . \quad \text { Q.E.D. }
$$

\section{Examples.}

7. We will show that the upper bound of Theorem 2 is the best possible, i.e. for each $n$, there exists a knot of enclosure genus $n$ which also has nullity equal to $n$.

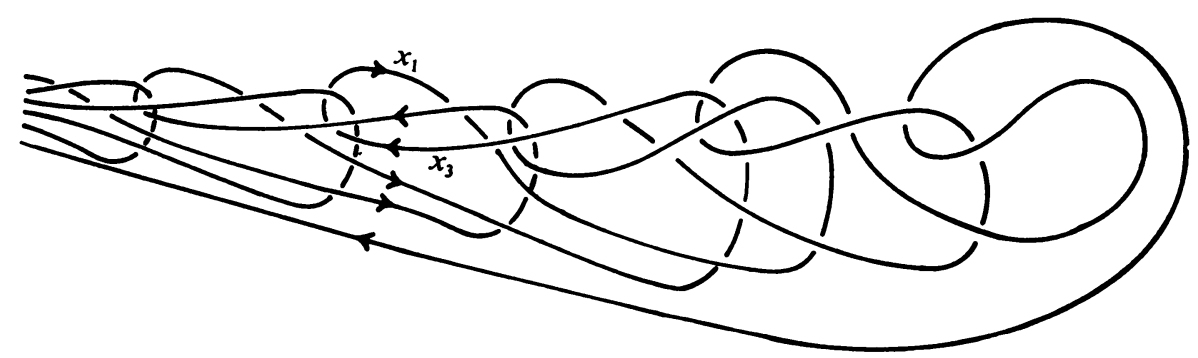

FIGURE 1 
We consider a variation of the "remarkable simple closed curve" of Fox [7]; instead of passing the arc $b_{i}$ of Figure 1 of [7] over just one tongue of the knot, we pass it over $n$ tongues. A projection of the knot so obtained when $n=2$ is shown in Figure 1, and it is easy to see that $k_{2}$ has enclosure genus at most equal to three. In general, $e\left(k_{n}\right) \leqq n+1$. We will show that the Alexander module of $k_{n}$ has nullity equal to $n+1$, thus showing that $e\left(k_{n}\right)=n+1$. Further, because $e(k) \leqq P(k, w) / 2$ for all knots $k$, we see that $P\left(k_{n}, w\right)=2 n+2$.

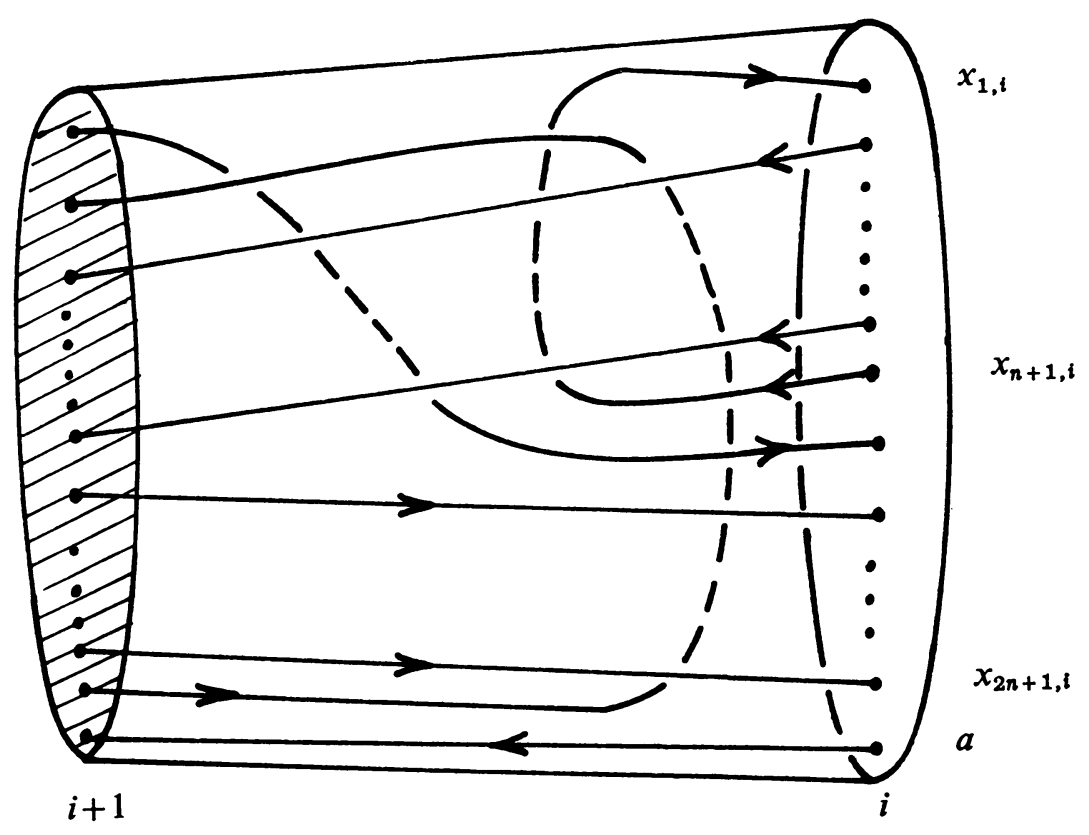

Figure 2

Using the techniques of [6], $k_{n}$ can be constructed from an infinite family of cylinders of the type shown in Figure 2. Then $\pi_{1}\left(S-k_{n}\right)$ has a presentation of the form

$$
\left(\bigcup_{i=0}\left\{x_{1, i}, x_{2, i}, \ldots, x_{2 n+1, i}, x_{2 n+2, i}=a\right\} ; r_{0}, r_{1}, \ldots\right)
$$

where $\boldsymbol{r}_{0}$ is the set

$\left(\alpha_{0}\right) x_{l, 0}=x_{2 n+3-l, 0}, l=1,2, \ldots, n+1$, and $\boldsymbol{r}_{i}$ consists of the following relations $(i=0,1,2, \ldots)$ :

(1 $i_{i, i+1} x_{2, i+1} \cdots x_{n+1, i+1}=x_{2, i+1} \cdots x_{n+1, i+1} x_{n+2, i}$

(2i $x_{2, i+1} \cdots x_{n+1, i+1} x_{n+1, i}=x_{1, i} x_{2, i+1} \cdots x_{n+1, i+1}$,

(3i $x_{2 n+1, i+1} W_{i}=W_{i} x_{2, i+1}$, where $W_{i}=x_{2 n+1, i} \cdots x_{n+2, i} \bar{x}_{n+1, i} \cdots \bar{x}_{2, i}$,

(4) $x_{l, i+1}=x_{l-1, i}, l=3, \ldots, n+1$,

(5i) $x_{n+l, i+1}=x_{n+l+1, i}, l=2, \ldots, n$,

(6) $x_{2 n+2, i}=a=W_{i} x_{1 i}$ (relation about the vertex). 
Let $\left(\alpha_{i}\right)$ be a set of relations of the form

$$
x_{l, i}=x_{2 n+3-l, i}, \quad l=1,2, \ldots, n+1 .
$$

Then if $\left(\alpha_{i}\right)$ and $\boldsymbol{r}_{i}$ are true, so are $\left(\alpha_{i+1}\right)$; since $\left(\alpha_{0}\right)$ holds, $\left(\alpha_{i}\right)$ is true for all $i$. Using this, I calculate that $\pi_{1}\left(S-k_{n}\right)$ has a presentation of the form

$$
\left(\left\{x_{2,0}, x_{3,0}, \ldots, x_{n+1,0}\right\} \cup \bigcup_{i=1}\left\{x_{2, i}\right\} ;\left(\beta_{i}\right)\right)
$$

where for $i \geqq n-1,\left(\beta_{i}\right)$ is a relation of the form

$$
\begin{aligned}
& x_{2, i+1} \cdots x_{2, i+2-n} x_{2, i+1-n} \bar{x}_{2, i+2-n} \cdots \bar{x}_{2, i+1} \\
& =x_{2, i+2} \cdots x_{2, i+3-n} x_{2, i+2-n} \bar{x}_{2, i+3-n} \cdots \bar{x}_{2, i+2}
\end{aligned}
$$

with a corresponding form for $i<n-1$. The Alexander matrix computed from this presentation has the infinite form shown in Figure 3, which has nullity $n+1$, as required.

$$
\left[\begin{array}{cccccccc}
1-t & -(t-1)^{2} & -t(t-1)^{2} & \cdots & t^{n-1}(2 t-1) & -t^{n} & 0 & 0 \\
0 & 1-t & -(t-1)^{2} & \cdots & -t^{n-2}(t-1)^{2} & t^{n-1}(2 t-1) & -t^{n} & 0 \\
0 & 0 & 1-t & \cdots & -t^{n-3}(t-1)^{2} & -t^{n-2}(t-1)^{2} & t^{n-1}(2 t-1) & -t^{n} \\
* & * & * & * & * & * & * & *
\end{array}\right]
$$

\section{FIGURE 3}

8. Let $k$ be a knot or an arc with one wild points $w$. Then the penetration index of $k$ at $w$, relative to surfaces of genus $r$, (cf. [2]), is the smallest integer $n$ such that there exist arbitrarily small neighbourhoods of $w$ which are handlebodies of genus $r$, each meeting $k$ on its boundary in $n$ points. We will denote this penetration index by $P_{r}(k, w)$.

(8.1) Let $H_{0} \supset H_{1} \supset \cdots$ be an admissible sequence for $k$ of minimal genus, and let $E$ be a 3-cell neighbourhood of $w$. Then there exists an index $N$ such that only one handle of $H_{N}$ meets $\mathrm{Bd} E$.

Proof. $k \cap E$ consists of two arcs from $\mathrm{Bd} E$ to $w$, and several arcs joining the points of $k \cap \operatorname{Bd} E$. Let $\delta$ be the minimum distance from $w$ to one of these arcs, and choose a 3-cell neighbourhood of $w, E^{\prime}$ say, which is centred at $w$ and is of radius less than $\delta$. Then only one arc $\alpha$ of $k \cap \mathrm{Cl}\left(S-E^{\prime}\right)$ meets the boundary of $E$.

We may construct a handlebody $H$ by adjoining to $E^{\prime}$ a tubular neighbourhood of this arc $\alpha$, and handles about the other arcs of $k$ lying in Int $(E)-E^{\prime}$. Since $\cap H_{i}=k$, there exists an index $N$ such that $H_{N} \subset$ Int $H$. We put $H_{N}$ and $\mathrm{Bd} E$ into general position so that $H_{N} \cap \mathrm{Bd} E$ is a family of discs on $\mathrm{Bd} E$. Each of these discs contains at most one point of $k$, so all but one handle of $H_{N}$ lies in the interior of $E$.

THEOREM 3. Let $k=k_{1} \cup k_{2}$, where $k_{i}$ is an arc with endpoint $w$. If

$$
r_{i}=\min \left\{n: P_{n}\left(k_{i}\right)=1\right\},
$$

then $e\left(k_{1} \cup k_{2}\right)>\max \left(r_{1}, r_{2}\right)$. 
Proof. Suppose $r_{1} \geqq r_{2}$, and let $E$ be a 3-cell neighbourhood of $w$. Then let $k_{1}^{\prime}$ be the subarc of $k_{1}$ which joins $k_{1} \cap \mathrm{Bd} E$ to $w$ in the interior of $E$. $k_{1}^{\prime}$ is of the same local type as $k_{1}$.

Let $H_{0} \supset H_{1} \supset \ldots$ be an admissible sequence for $k$ of genus $e(k)$. Then there exists an index $N$ such that only one handle of $H_{N}$ meets $\mathrm{Bd} E$. We put $H_{N}$ and $\mathrm{Bd} E$ into general position so that $H_{N} \cap \mathrm{Bd} E$ is a family of discs on $\mathrm{Bd} E$, and from the proof of (8.1) we may assume that each of these discs meets $k$ in at most one point. Then the component of $\mathrm{Cl}\left(H_{N}-H_{N} \cap \mathrm{Bd} E\right)$ which contains $w$ is a handlebody $H_{N}^{*}$ of genus $e(k)-1$ which contains only one point of $k_{1}^{\prime}$ on its boundary.

We take another 3-cell $E^{\prime} \subset$ Int $H_{N}$, and construct a handlebody $H^{\prime}$ as in the proof of (8.1); we may assume $H^{\prime} \subset$ Int $H_{N}$. There exists an index $M$ such that $H_{M} \subset$ Int $H^{\prime}$, and proceeding as above we obtain a handlebody $H_{M}^{*} \subset H_{N}^{*}$ of genus $e(k)-1$ meeting $k_{1}^{\prime}$ on its boundary in only one point.

So we construct a sequence $H_{N}^{*} \supset H_{M}^{*} \supset \ldots$ which contains arbitrarily small handlebodies of genus $e(k)-1$ which meet $k_{1}^{\prime}$ on their boundaries in one point. That is, $e(k)-1 \geqq r_{1}$. Q.E.D.

Corollary. Let $k$ be an arc which is wild at one endpoint, with $P_{r}=1$ and $P_{r-1}>1$. Then the "mixed composite" of the trivial knot $k_{0}$ with respect to $k[8$, p. 336] has enclosure genus equal to $r+1$.

Proof. The mixed composite $k^{*}$ is really the union of two copies of the arc $k$, so $e\left(k^{*}\right) \geqq r+1$. But one can construct an admissible sequence for $k^{*}$ from a sequence of arbitrarily small handlebodies of genus $r$, by sewing on an extra handle. Therefore, $e\left(k^{*}\right) \leqq r+1$.

To prove that the lower bound of Theorem 2 is the best possible, it is only necessary to produce a family of arcs $k_{n}$, such that $P_{n}\left(k_{n}\right)=1$ and $P_{n-1}\left(k_{n}\right)>1$. For then the mixed composite of $k_{0}$ with respect to $k_{n}$ has enclosure genus $n+1$; by V.B.6 of [8], there is an epimorphism from $\pi_{1}\left(S-k_{0}\right)=Z$ to $\pi_{1}\left(S-k_{n}^{*}\right)$, so the Alexander module of $\pi_{1}\left(S-k_{n}^{*}\right) \cong Z$ has unit nullity.

It would appear that the $\operatorname{arcs} A_{n}$ of Alford and Ball [1] have these properties, although I can find no proof of this.

RemarK. Our examples have all been "almost unknotted" in the sense of Fox [7], so this geometric property does not determine the nullity of a knot (cf. [3, §6]).

Added in proof. (i) There is a gap in the proof of Theorem 1, in that we must prove $H$ admits all coproducts over infinite families of $H$-groups, and that the functor $M$ preserves these coproducts. For the family $\left\{\left[G_{i}, \alpha_{i}\right], i \in I\right\}$, consider the coproduct $G=\oplus G_{i}$ in $G$, and define the map $\alpha: G \rightarrow H$ by $\alpha(g)=\prod_{i} \alpha_{i}(g)$. There is an obvious isomorphism from $M[G, \alpha]$ to $\oplus M\left[G_{i}, \alpha_{i}\right]$.

(ii) M. D. Taylor (doctoral dissertation, Florida State University, 1962) has exhibited a family of arcs $k_{n}$ with $P_{n}\left(k_{n}\right)=1$ and $P_{n-1}\left(k_{n}\right)>1$. So the lower bound of Theorem 2 is the best possible. 


\section{REFERENCES}

1. W. R. Alford and B. J. Ball, Some almost polyhedral wild arcs, Duke Math. J. 30 (1963), 33-38.

2. B. J. Ball, "Penetration indices and their applications" in Ropology of 3-manifolds, edited by M. K. Fort, Jr., Prentice-Hall, New York, 1962, pp. 37-39.

3. E. J. Brody, On infinitely generated modules, Quart. J. Math. Oxford Ser. 11 (1960), 141-150.

4. R. H. Crowell, On the van Kampen theorem, Pacific J. Math. 9 (1959), 43-50.

5. - Corresponding group and module sequences, Nagoya Math. J. 19 (1961), $27-40$.

6. R. H. Fox and E. Artin, Some wild cells and spheres in three-dimensional space, Ann. of Math. 49 (1948), 979-990.

7. R. H. Fox, A remarkable simple closed curve, Ann. of Math. 50 (1949), 264-265.

8. S. J. Lomonaco, An algebraic theory of local knottedness, Trans. Amer. Math. Soc. 129 (1967), 322-343.

9. S. MacLane, Homology, Springer-Verlag, New York, 1963.

10. B. Mitchell, Theory of categories, Academic Press, New York, 1965.

11. H. F. Trotter, Homology of group systems with applications to knot theory, Ann. of Math. 76 (1962), 464-498.

The University of New South Wales, Kensington, N.S.W., Australia

The UNIVERSITY OF Virginia, Charlottesville, Virginia 